\title{
Comparison of Uptake of Multiple Clinical Radiotracers into Brown Adipose Tissue Under Cold-Stimulated and Nonstimulated Conditions
}

\author{
Shingo Baba ${ }^{1}$, James M. Engles ${ }^{1}$, David L. Huso ${ }^{2}$, Takayoshi Ishimori ${ }^{1}$, and Richard L. Wahl ${ }^{1}$ \\ ${ }^{I}$ Division of Nuclear Medicine, Russell H. Morgan Department of Radiology and Radiological Science, Johns Hopkins Medical \\ Institutions, Baltimore, Maryland; and ${ }^{2}$ Department of Molecular and Comparative Pathology, Johns Hopkins Medical Institutions, \\ Baltimore, Maryland
}

\begin{abstract}
Our objective was to determine whether multiple clinically useful radiotracers accumulate in brown adipose tissue (BAT) and to assess their uptake in rats kept at room temperature or exposed to a cold environment. Methods: The following radiotracers were injected intravenously into groups of 6 female Wistar rats: ${ }^{201} \mathrm{TI}-$ chloride (TICl), ${ }^{123} \mathrm{I}-\mathrm{metaiodobenzylguanidine} \mathrm{(MIBG),}$ 99mTc-sestamibi (MIBI), ${ }^{18} \mathrm{~F}$ - or ${ }^{3} \mathrm{H}-\mathrm{FDG},{ }^{3} \mathrm{H}$-L-methionine, and ${ }^{3} \mathrm{H}-$
\end{abstract} thymidine. BAT-stimulated animals were maintained at $4^{\circ} \mathrm{C}$ for $4 \mathrm{~h}$ before tracer injection, whereas control animals were kept at approximately $22.5^{\circ} \mathrm{C}$. The animals were sacrificed at 20-60 min after tracer injection, and BAT, major organs, and blood were extracted, weighed, and measured for radioactivity. The localization of uncoupling protein-1, glucose transporter-1, and norepinephrine transporter was evaluated with immunohistochemical staining in both groups. Results: We determined the percentage injected dose (\%ID) per gram of each radiotracer in interscapular BAT, normalized to blood \%ID/g. In control animals, this uptake ratio $( \pm S D)$ was $8.44 \pm 3.39$ for ${ }^{201} \mathrm{TICl}, 9.77 \pm 6.06$ for ${ }^{123 /-}$ MIBG, $37.30 \pm 14.42$ for ${ }^{99 m T c-M I B I, ~} 5.47 \pm 4.44$ for ${ }^{18} \mathrm{~F}-$ or ${ }^{3} \mathrm{H}-F D G, 1.93 \pm 0.87$ for ${ }^{3} \mathrm{H}-\mathrm{L}-$ methionine, and $1.22 \pm 0.74$ for ${ }^{3} \mathrm{H}-$ thymidine. Compared with uptake at room temperature, uptake after exposure to cold increased 26.4 -fold $(P<0.01)$ for ${ }^{18} \mathrm{~F}$ - or ${ }^{3} \mathrm{H}-\mathrm{FDG}$ and increased significantly $(P<0.05)$ for ${ }^{201} \mathrm{TI}(2.04-$ fold), ${ }^{123}$ I-MIBG (3.25-fold), and ${ }^{3} \mathrm{H}$-L-methionine (3.11-fold). Immunohistochemical staining revealed increased glucose transporter-1 and norepinephrine transporter expression in BAT cell membranes and blood vessels after exposure to cold, whereas uncoupling protein-1 was expressed in the cytoplasm under both control and cold-stimulated conditions. Conclusion: BAT uptake of ${ }^{18} \mathrm{~F}-$ or ${ }^{3} \mathrm{H}-\mathrm{FDG},{ }^{123} \mathrm{I}-\mathrm{MIBG}$, and ${ }^{3} \mathrm{H}-\mathrm{L}-\mathrm{methionine}$ was significantly increased over the control state by exposure to cold. Increased uptake of ${ }^{201} \mathrm{TICl}$ relative to blood in cold-stimulated BAT suggests that blood flow in BAT is increased by exposure to cold. The greater increased uptake with ${ }^{18} \mathrm{~F}$ - or ${ }^{3} \mathrm{H}-\mathrm{FDG},{ }^{123} \mathrm{I}-\mathrm{MIBG}$, and ${ }^{3} \mathrm{H}-\mathrm{L}-$ methionine, and the immunohistostaining findings, suggest that other factors in addition to blood flow (e.g., increased metabolism, increased transport, or metabolic trapping of the tracers) are in-

\footnotetext{
Received Mar. 14, 2007; revision accepted Jun. 27, 2007.

For correspondence or reprints contact: Richard L. Wahl, MD, Division of Nuclear Medicine, Department of Radiology, Johns Hopkins Medical Institutions, 601 N. Caroline St., Room 3223A, Baltimore, MD 21287.

E-mail: rwahl@jhmi.edu

COPYRIGHT @ 2007 by the Society of Nuclear Medicine, Inc.
}

volved in cold-stimulated BAT activation. Knowledge that high uptake in BAT may possibly be observed on clinical scans using several radiotracers, especially after patients are exposed to the cold, may lead to more accurate interpretation of clinical studies.

Key Words: BAT; cold exposure; FDG; MIBG; methionine

J Nucl Med 2007; 48:1715-1723

DOI: 10.2967/jnumed.107.041715

B rown adipose tissue (BAT) differs from white adipose tissue morphologically. BAT is so named because of the visible brownish color resulting from its rich vascularization and increased mitochondrial content. BAT is generally located in deep cervical regions, including the supraclavicular area, the interscapular and paravertebral regions, and areas near large blood vessels. It is characterized by a unique metabolic pathway that results in the generation of heat. This heat generation by BAT is initiated by activation of the sympathetic nervous system, especially via activation of the $\beta 3$-adrenergic receptor. Signals of decreasing body temperature, recent feeding, and high reserves of body energy have been reported to be the initial triggers that stimulate BAT (1).

BAT has recently received considerable attention in clinical nuclear medicine (2). High uptake of ${ }^{18} \mathrm{~F}-\mathrm{FDG}$ in nonmalignant supraclavicular, thoracic costovertebral, and upper abdominal regions has been explained as uptake in BAT $(3,4)$. This high uptake may sometimes be indistinguishable from uptake in tumors or lymph nodes. Increased uptake in the supraclavicular region has been reported to occur in $2.5 \%-4.0 \%$ of patients undergoing ${ }^{18} \mathrm{~F}-\mathrm{FDG}$ PET/CT studies (5), is more common in women, and in some reports is more common in winter $(3,5)$. There is also evidence that ${ }^{18} \mathrm{~F}$-FDG uptake is reduced by controlling environmental temperature before a PET scan (6). Understanding the function of BAT and the influence of exposure to cold on BAT activity is potentially important for the interpretation of ${ }^{18} \mathrm{~F}-\mathrm{FDG}$ PET/CT studies.

High tracer uptake in BAT has been reported for clinical radiotracers other than ${ }^{18} \mathrm{~F}$-FDG. Okuyama et al. demonstrated 
that ${ }^{123}$ I-metaiodobenzylguanidine (MIBG) accumulated in the BAT of experimental animals and that ${ }^{123} \mathrm{I}-\mathrm{MIBG}$ uptake in these regions in children occurred more often in cold weather than in warm weather $(7,8)$. These results are consistent with the hypothesis that the increased incidence of high radiotracer uptake in BAT is related to activation of BAT. Fukuchi et al. reported increased uptake of ${ }^{99 \mathrm{~m}} \mathrm{Tc}$-tetrofosmin in BAT in pediatric patients (9). Higuchi et al. described a patient who displayed high ${ }^{99 \mathrm{~m}} \mathrm{Tc}$-sestamibi (MIBI) uptake in BAT but no uptake of ${ }^{201} \mathrm{Tl}$-chloride $(\mathrm{TlCl})$ in a dual-tracer SPECT study (10). These studies indicate that $99 \mathrm{~m}$ Tc-labeled agents for cardiac perfusion may also reflect BAT activity, especially its rich mitochondrial function. Our current study originated from the question of what happens to BAT uptake when common SPECT and PET radiotracers are used in animals at room temperature (approximately $22^{\circ} \mathrm{C}-23^{\circ} \mathrm{C}$ ) or under cold conditions.

BAT is known to be stimulated by physiologic or pharmacologic intervention $(11,12)$, and activation of BAT may result in an increase in radiotracer uptake. This uptake may represent the metabolic activity of BAT. However, it is still unknown which radiotracers accumulate in BAT and how this uptake will be changed by BAT activation.

We previously reported that uptake of ${ }^{18} \mathrm{~F}-\mathrm{FDG}$ in BAT can be increased by a short period of exposure to cold $\left(4^{\circ} \mathrm{C}\right.$ for $4 \mathrm{~h}$ ), suggesting that the procedure is suitable for a simple BAT-activation model (11). The purpose of this study was to systematically evaluate the BAT uptake of various clinically useful radiotracers using a cold-activation method in rodents. We also evaluated the degree of expression and the localization of uncoupling protein-1 (UCP-1), glucose transporter-1 (Glut-1), and norepinephrine transporter (NET) by immunohistostaining. An awareness of the distribution of commonly used clinical radiotracers in BAT, normally and after exposure to cold, should help avoid errors in the interpretation of various nuclear medicine studies.

\section{MATERIALS AND METHODS}

\section{Animals}

Eight- to 12-wk-old female Wistar rats (mean body weight, 225 g) were used in this study and were kept in the animal housing facilities at least $1 \mathrm{wk}$ before the start of experimentation. Food and water were given ad libitum. The study was performed as part of a project approved by the animal research committee at the Johns Hopkins Medical Institutions.

\section{BAT Activation}

To stimulate BAT activity, we maintained the experimental animals at $4^{\circ} \mathrm{C}$ for $4 \mathrm{~h}$ before tracer injection, whereas control animals were kept at room temperature (approximately $22^{\circ} \mathrm{C}-$ $\left.23^{\circ} \mathrm{C}\right)$. We chose this condition $\left(4^{\circ} \mathrm{C}\right.$ for $\left.4 \mathrm{~h}\right)$ according to previous reports to ensure that the BAT of all animals was stimulated adequately and reproducibly $(1,11,13)$. To avoid the effect of anesthesia on BAT activity, no anesthetic agent was used in any procedure throughout the study except for euthanasia.

\section{Biodistribution Study}

Tracers were injected intravenously into groups of 6 rats each. The uptake time was $20 \mathrm{~min}$ for ${ }^{201} \mathrm{TlCl}, 20 \mathrm{~min}$ for ${ }^{123} \mathrm{I}-\mathrm{MIBG}$, $60 \mathrm{~min}$ for ${ }^{99 \mathrm{~m} T c-M I B I}, 60 \mathrm{~min}$ for ${ }^{18} \mathrm{~F}-$ or ${ }^{3} \mathrm{H}-\mathrm{FDG}, 60 \mathrm{~min}$ for ${ }^{3} \mathrm{H}$-L-methionine, and $20 \mathrm{~min}$ for ${ }^{3} \mathrm{H}$-thymidine. A dose of 7,400 $\mathrm{kBq}(200 \mu \mathrm{Ci})$ and $185 \mathrm{kBq}(5 \mu \mathrm{Ci})$ per animal was injected for $\gamma$-emitters and tritium-labeled tracers, respectively. In some experimental groups, $\gamma$-emitting and tritium-labeled isotopes were simultaneously given by dual injection (e.g., ${ }^{18} \mathrm{~F}-\mathrm{FDG}$ plus ${ }^{3} \mathrm{H}-\mathrm{L}-$ methionine, or ${ }^{99 \mathrm{~m}} \mathrm{Tc}-\mathrm{MIBI}$ plus $\left.{ }^{3} \mathrm{H}-\mathrm{FDG}\right)$. Both $\gamma$-emitters and tritium-labeled tracers were used in the FDG group. Animals in the cold-exposure group were kept in the cold after tracer injection. After the uptake time appropriate for each tracer, the animals were sacrificed; BAT, major organs, and blood were extracted and weighed; and tissue radioactivity was measured with a $\gamma$-counter. For the dual-tracer injection groups, tissue samples were then dissolved in solution (Solvable; Perkins Elmer) and the radioactivity of tritium was measured using a liquid scintillation counter. To avoid contamination from the $\gamma$-radiotracer, the tritium samples were counted after at least 20 half-lives of the coinjected $\gamma$-emitter.

The percentage injected dose (\%ID) per gram of tissue normalized by kilogram of body weight $(\% \mathrm{ID} / \mathrm{g} \times \mathrm{kg}[\% \mathrm{ID} / \mathrm{body}$ weight]), as well as tissue $\% \mathrm{ID} / \mathrm{g}$ standardized by $\% \mathrm{ID} / \mathrm{g}$ of blood (\%ID/blood) and by \%ID/g of muscle (\%ID/muscle), was calculated and compared among tissues. Except for cold activation, all procedures including radiotracer injection were performed at room temperature (approximately $22^{\circ} \mathrm{C}-23^{\circ} \mathrm{C}$ ) in a climate-controlled room.

\section{Immunohistostaining}

To evaluate cold-induced histologic changes of BAT, histologic (hematoxylin-eosin staining) and immunohistochemical evaluations were performed on samples obtained from animals under the control and the cold-stimulated conditions. The rabbit antimitochondrial UCP-1 antibody, anti-Glut-1 antibody, and anti-NET antibody were used for immunohistochemical evaluation. Antibody staining was performed using the avidin-biotin-peroxidase (avidin-biotin complex) method on dewaxed 5- $\mu$ m-thick sections from a formalin-fixed, paraffin-embedded sample. The details of this procedure have been described elsewhere (14). The polyclonal rabbit anti-UCP-1 antibody (Sigma), the polyclonal rabbit antiGlut-1 antibody (Chemicon), and the polyclonal rabbit anti-NET antibody (Chemicon) were used as primary antibodies. Each of these antibodies was used in a 1:300 dilution in separate studies. Samples were reviewed by light microscopy to determine the presence and location of these proteins.

\section{Statistical Analysis}

The data for the cold-stimulated group (\%ID/body weight, $\% \mathrm{ID} / \mathrm{blood}$, and \%ID/muscle) were compared with the data for the control group using the Mann-Whitney $U$ test. A $P$ value of less than 0.05 was considered statistically significant.

\section{RESULTS}

Interscapular BAT was easily located in each rat at the time of dissection. Interscapular BAT in the cold-exposure group was reddish brown without exception on direct visual inspection, whereas interscapular BAT in the control group was whitish brown (Fig. 1A). On staining of interscapular 


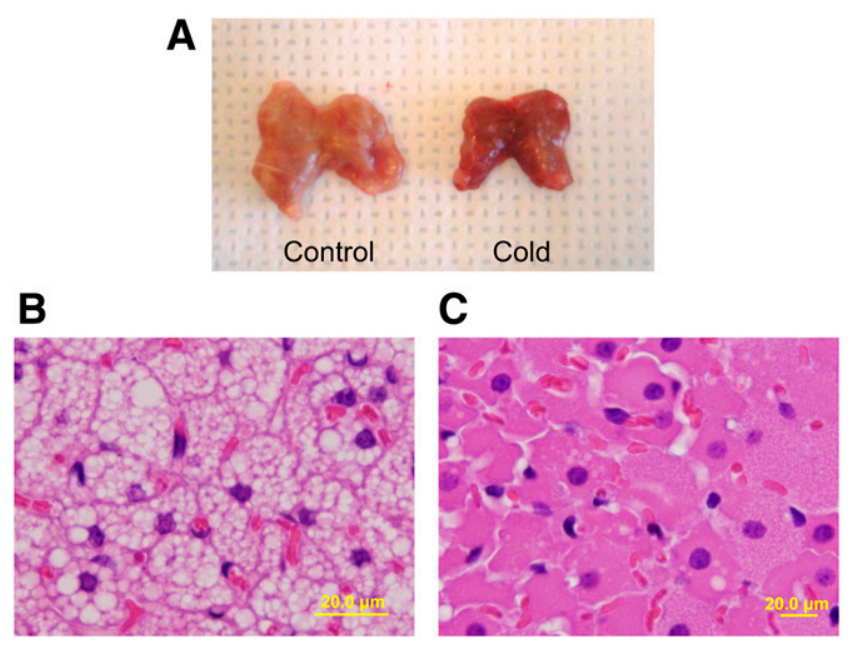

FIGURE 1. (A) Resected interscapular BAT after $4 \mathrm{~h}$ of exposure to cold (right) becomes darker and redder than control BAT kept at room temperature (left). (B and C) On light microscopy, major structural changes are seen in BAT cells stained with hematoxylin-eosin. Many large lipid vacuoles are seen in control BAT cells (B), but vacuoles are almost nonexistent in BAT cells exposed to cold (C). Effect of exposure to cold is grossly visible.

BAT with hematoxylin-eosin, numerous lipid vacuoles were seen in the BAT cells from the control animals (Fig. 1B), but most of these lipid vacuoles were not present in the animals exposed to cold (Fig. 1C).

$\%$ ID/blood is summarized in Figure 2A. Under control conditions, ${ }^{99 \mathrm{~m} T c-M I B I}$ had the highest uptake among these tracers. \%ID/blood was lower for ${ }^{18} \mathrm{~F}-$ or ${ }^{3} \mathrm{H}-\mathrm{FDG}$ than for ${ }^{201} \mathrm{TlCl},{ }^{123} \mathrm{I}-\mathrm{MIBG}$, and ${ }^{99 \mathrm{~m}} \mathrm{Tc}-\mathrm{MIBI}$ under control conditions. After stimulation by cold, \%ID/blood was increased, compared with \%ID/blood under nonstimulated conditions: 2.04-fold for ${ }^{201} \mathrm{TlCl}, 3.25$-fold for ${ }^{123} \mathrm{I}$-MIBG, 1.39-fold for ${ }^{99 \mathrm{~m} T c-M I B I}, 26.4$-fold for ${ }^{18} \mathrm{~F}$ - or ${ }^{3} \mathrm{H}-\mathrm{FDG}, 3.11$-fold for ${ }^{3} \mathrm{H}$-L-methionine, and 2.17-fold for ${ }^{3} \mathrm{H}$-thymidine (Fig. 2A). A statistically significant $(P<0.05)$ increase was seen for ${ }^{201} \mathrm{TlCl},{ }^{123} \mathrm{I}-\mathrm{MIBG},{ }^{18} \mathrm{~F}-$ or ${ }^{3} \mathrm{H}-\mathrm{FDG}$, and ${ }^{3} \mathrm{H}-\mathrm{L}-\mathrm{methionine}$. BAT uptake of ${ }^{99 \mathrm{~m} T c-M I B I}$ tended to increase with exposure to cold $(P=0.07)$. Uptake of ${ }^{3} \mathrm{H}$-thymidine did not change significantly $(P=0.17)$. Changes in $\% \mathrm{ID} /$ blood for other major organs are summarized in Table 1. Changes occurred in brain, stomach, and spleen (decreased uptake with ${ }^{18} \mathrm{~F}$ - or ${ }^{3} \mathrm{H}-\mathrm{FDG}$ ); white adipose tissue (increased uptake with ${ }^{3} \mathrm{H}-\mathrm{L}-$ methionine); and kidney (increased uptake with ${ }^{99 \mathrm{~m}} \mathrm{Tc}$ MIBI).

$\% \mathrm{ID} /$ muscle and $\% \mathrm{ID} /$ body weight are summarized in Figures $2 \mathrm{~B}$ and $2 \mathrm{C}$, respectively.

Under control conditions, ${ }^{123}$ I-MIBG had the highest value for $\% \mathrm{ID} /$ body weight among the tracers. Uptake of ${ }^{18} \mathrm{~F}$ - or ${ }^{3} \mathrm{H}-\mathrm{FDG}$ was relatively low among the tracers under control conditions. After stimulation by cold, statistically significant $(P<0.05)$ increases were seen for ${ }^{123} \mathrm{I}-\mathrm{MIBG},{ }^{18} \mathrm{~F}-$ or ${ }^{3} \mathrm{H}-$ FDG, and ${ }^{3} \mathrm{H}-\mathrm{L}-\mathrm{methionine}$. No significant differences in uptake were seen between ${ }^{18} \mathrm{~F}$-FDG and ${ }^{3} \mathrm{H}$-FDG (data not

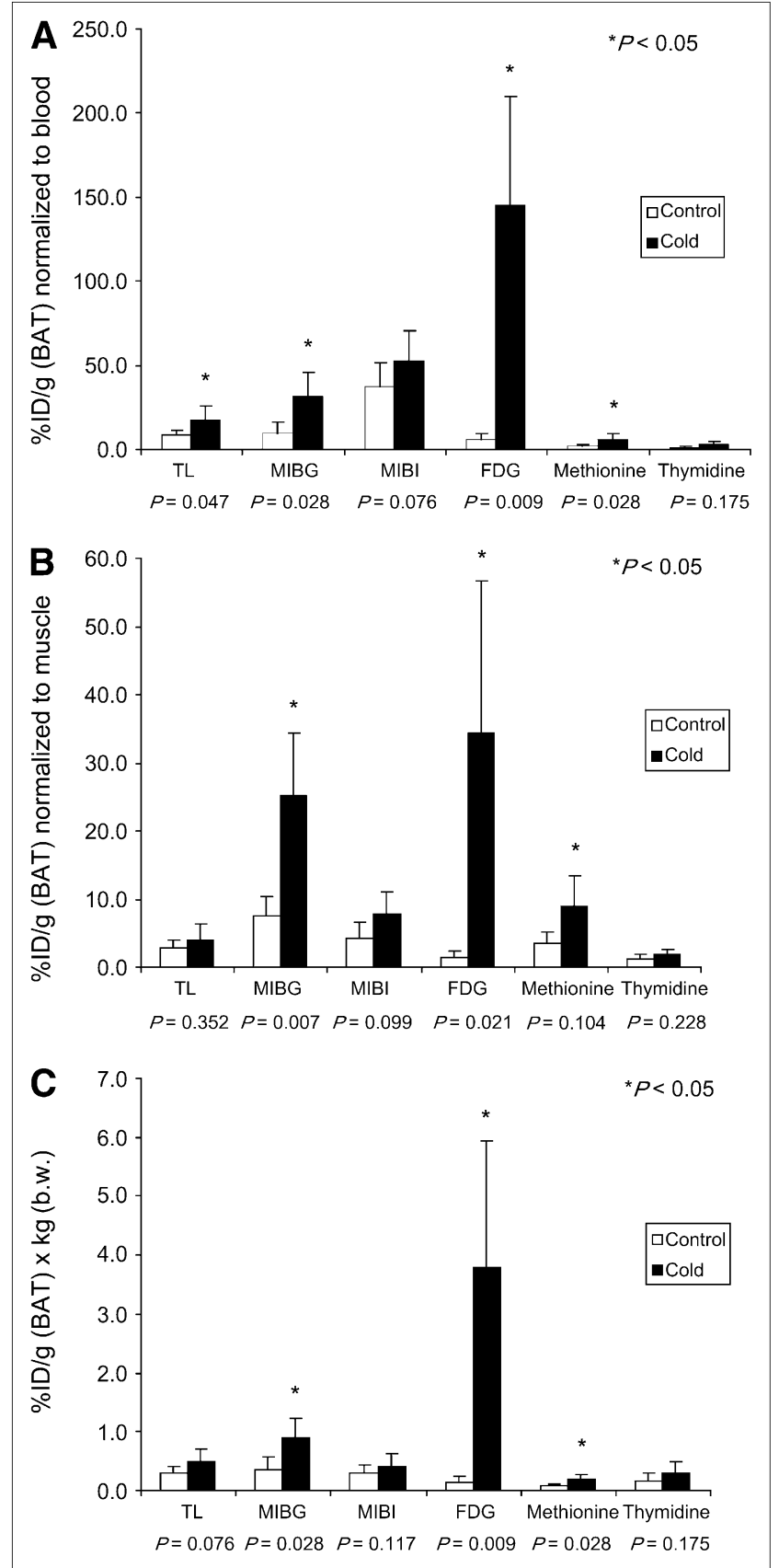

FIGURE 2. \%ID/blood (A), \%ID/muscle (B), and \%ID/body weight (\%ID/g $\times \mathrm{kg}$ [b.w.]) (C) for various tracers in interscapular BAT of control rats (white) and cold-stimulated rats (black). Under control conditions, highest uptake was seen in ${ }^{123}$ I-MIBG or $99 \mathrm{mTc}-\mathrm{MIBI}$. After cold activation, average uptake increased for all tracers. A, B, and C show statistically significant increases $(P<0.05)$ in tracer uptake in rats injected with ${ }^{123}$ MIBG, ${ }^{18} \mathrm{~F}-$ or ${ }^{3} \mathrm{H}-\mathrm{FDG}$, and ${ }^{3} \mathrm{H}-\mathrm{L}-$ methionine. Increase in uptake of ${ }^{201} \mathrm{TICl}$, compared with control, was also significant when normalized to blood.

shown). Changes in $\% \mathrm{ID} /$ muscle and \%ID/body weight for other major organs are summarized in Tables 2 and 3, respectively. Changes occurred in the stomach and spleen (decreased uptake with ${ }^{18} \mathrm{~F}$ - or ${ }^{3} \mathrm{H}-\mathrm{FDG}$ ) and in the liver (increased uptake with ${ }^{3} \mathrm{H}$-L-methionine). 


\begin{tabular}{|c|c|c|c|c|c|c|c|}
\hline \multirow{12}{*}{ 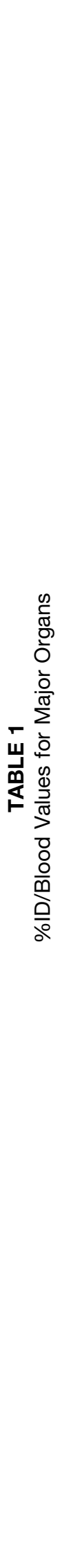 } & 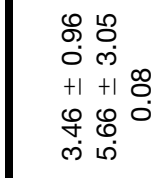 & 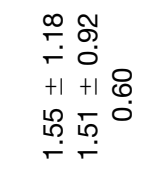 & 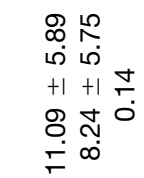 & 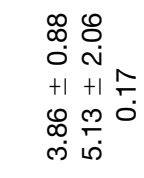 & 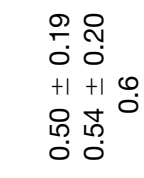 & 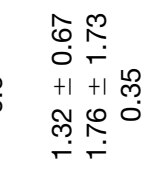 & \\
\hline & 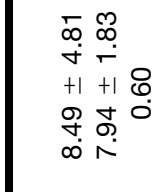 & 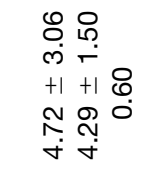 & 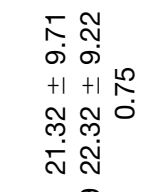 & 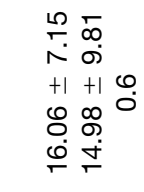 & 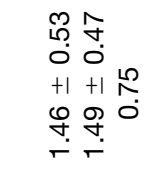 & 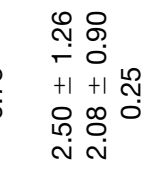 & \\
\hline & 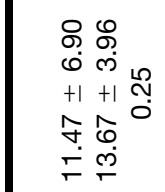 & 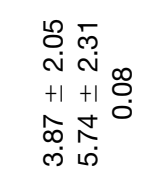 & 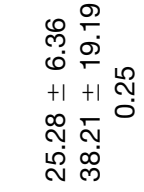 & 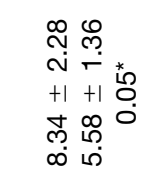 & 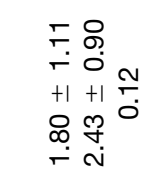 & 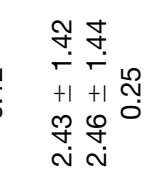 & \\
\hline & 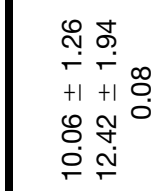 & 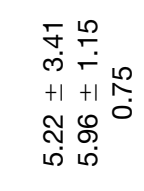 & 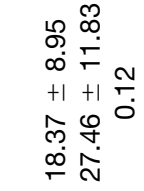 & 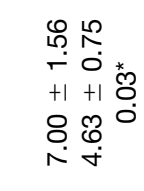 & 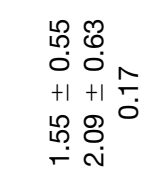 & 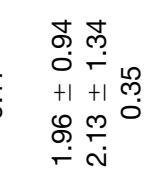 & \\
\hline & 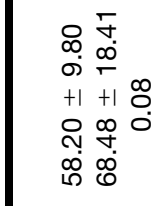 & 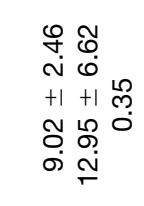 & 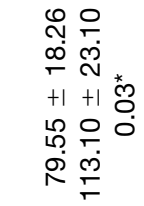 & 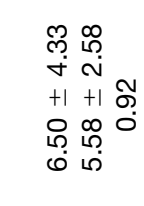 & 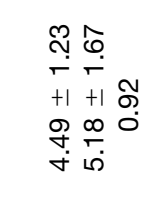 & 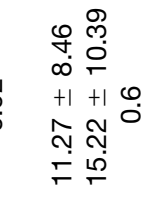 & \\
\hline & 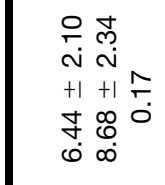 & 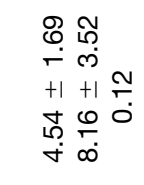 & 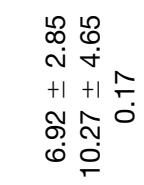 & 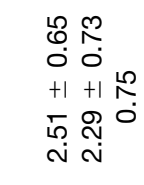 & 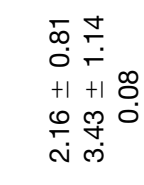 & 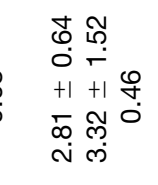 & \\
\hline & 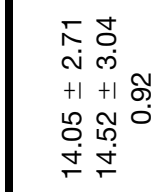 & 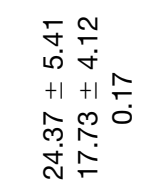 & 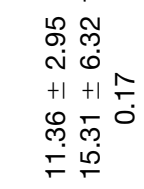 & 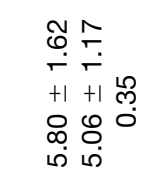 & 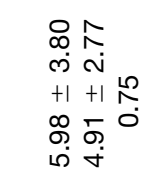 & 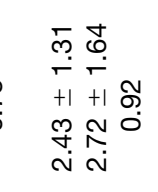 & \\
\hline & 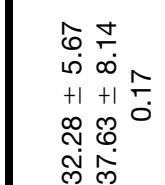 & 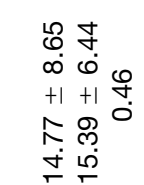 & 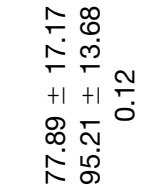 & 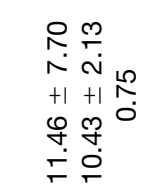 & 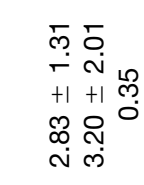 & 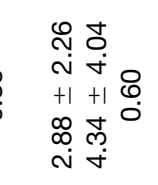 & \\
\hline & 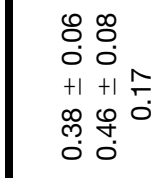 & 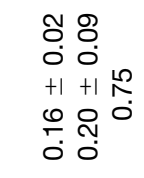 & 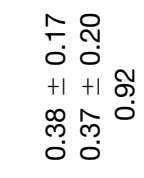 & 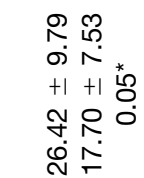 & 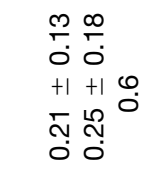 & 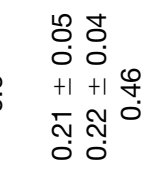 & \\
\hline & 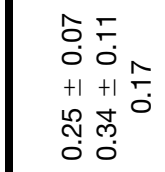 & 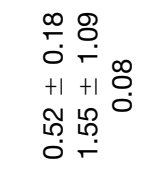 & 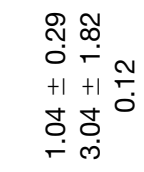 & 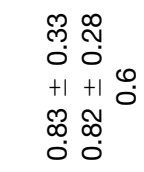 & 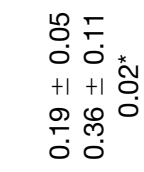 & 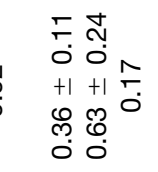 & \\
\hline & 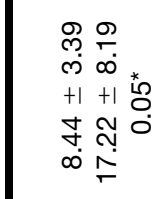 & 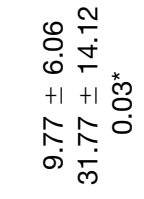 & 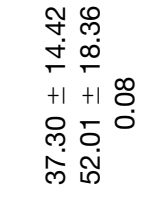 & 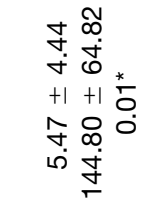 & 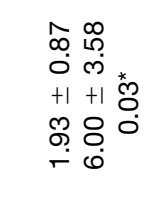 & 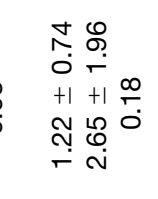 & 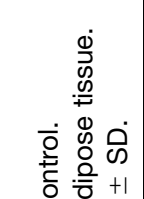 \\
\hline & 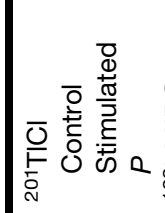 & 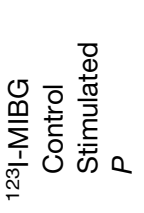 & 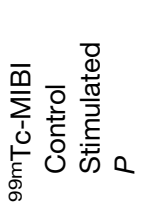 & 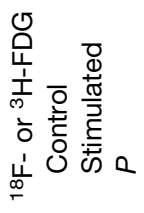 & 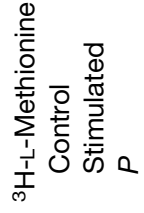 & 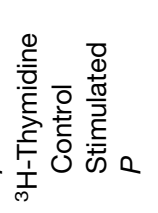 & 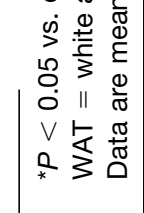 \\
\hline
\end{tabular}




\begin{tabular}{|c|c|c|c|c|c|c|c|c|}
\hline \multirow{12}{*}{ 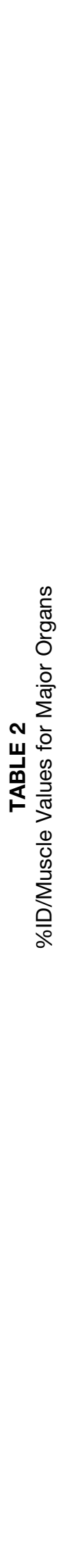 } & $\begin{array}{l}\frac{8}{0} \\
\frac{\circ}{0}\end{array}$ & 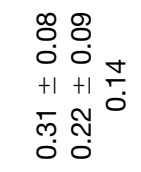 & 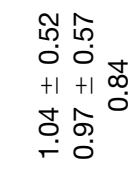 & 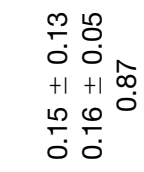 & 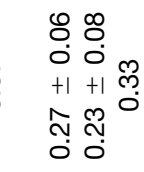 & 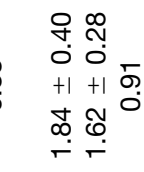 & $\begin{array}{ll}\hat{m} & 0 \\
0 & 0 \\
0 & 0 \\
+1 & +1 \\
0 & 0 \\
0 & 0 \\
0 & 0 \\
0 & 0\end{array}$ & \\
\hline & $\frac{\tau}{0}$ & 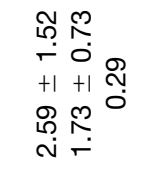 & 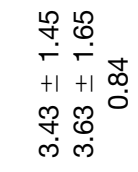 & 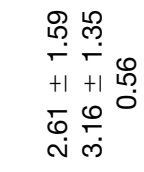 & 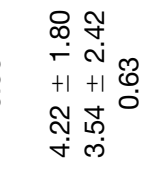 & 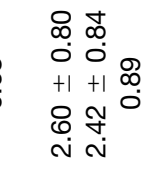 & 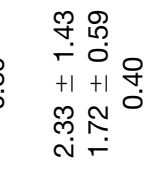 & \\
\hline & 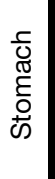 & 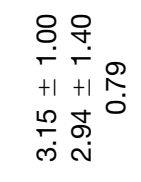 & 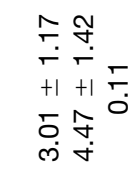 & 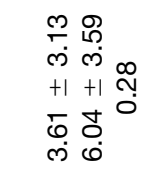 & 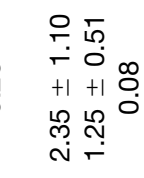 & 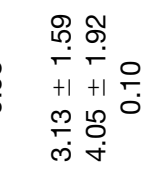 & 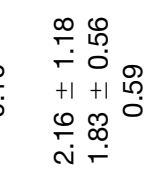 & \\
\hline & $\begin{array}{l}\frac{c}{\Phi} \\
\frac{\Phi}{0} \\
\mathcal{C}\end{array}$ & 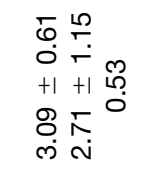 & 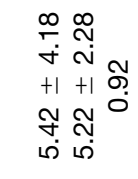 & 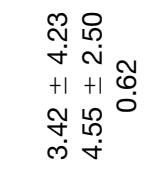 & 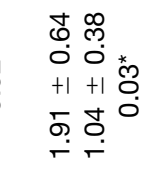 & 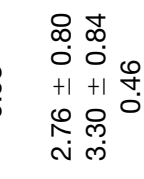 & 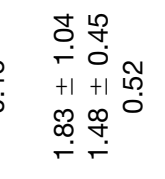 & \\
\hline & $\begin{array}{l}\vec{d} \\
\frac{\bar{c}}{\overline{0}} \\
\frac{\bar{y}}{2}\end{array}$ & 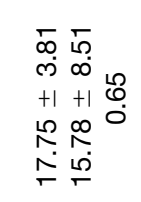 & 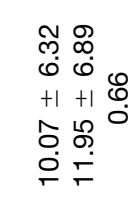 & 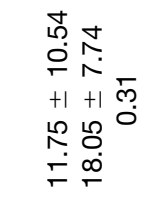 & 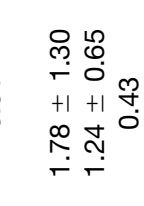 & 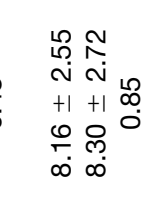 & 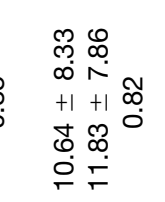 & \\
\hline & $\stackrel{\grave{\Phi}}{\stackrel{亠}{\Xi}}$ & 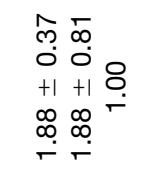 & 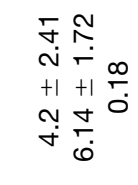 & 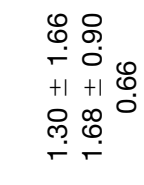 & 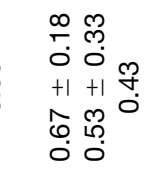 & 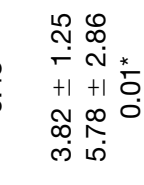 & 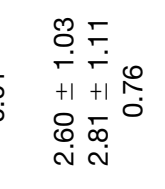 & \\
\hline & $\stackrel{\overparen{\Xi}}{\Xi}$ & 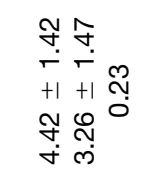 & 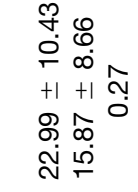 & 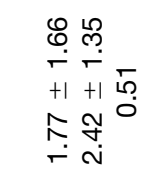 & 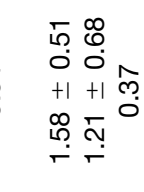 & 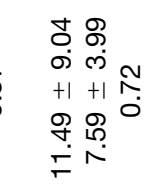 & 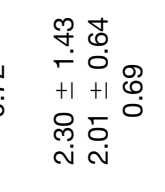 & \\
\hline & $\begin{array}{l}\frac{T}{\mathbb{\varpi}} \\
\frac{\mathbb{\Phi}}{I}\end{array}$ & 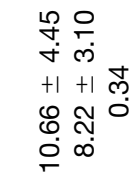 & 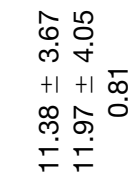 & 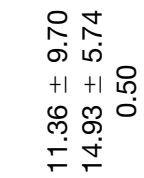 & 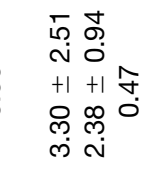 & 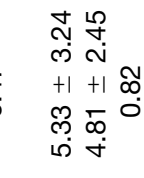 & 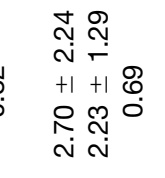 & \\
\hline & 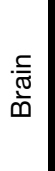 & 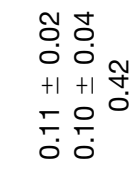 & 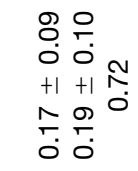 & 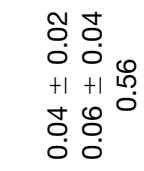 & 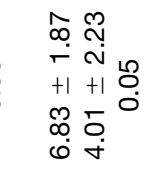 & 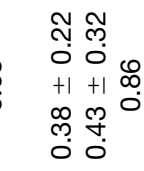 & 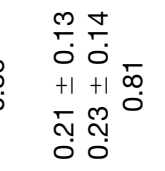 & \\
\hline & 攴 & $\begin{array}{ll}5 & 0 \\
0 & 0 \\
0 & 0 \\
+1 & 1 \\
+1 & 0 \\
0 & 0 \\
0 & 0 \\
0 & 0 \\
0 & 0\end{array}$ & 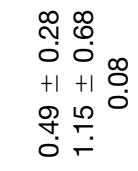 & 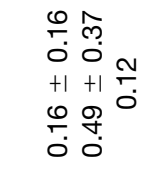 & 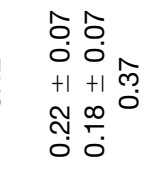 & 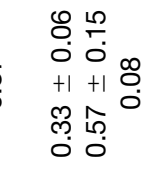 & 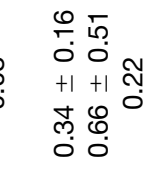 & \\
\hline & 卓 & 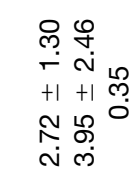 & 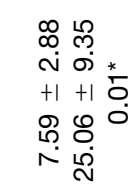 & 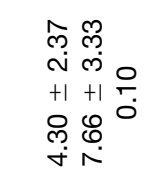 & 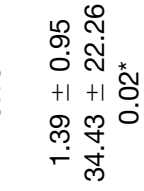 & 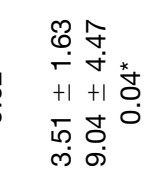 & 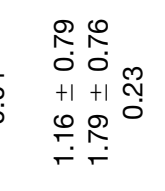 & 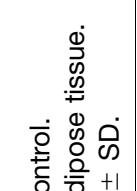 \\
\hline & 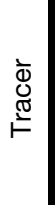 & 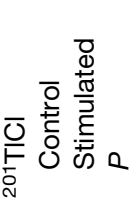 & 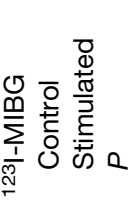 & 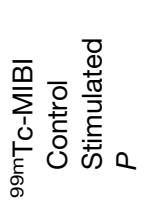 & 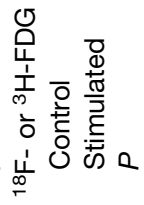 & 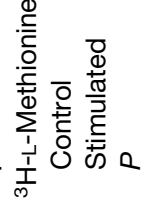 & 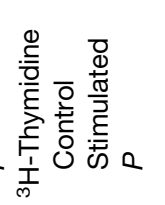 & 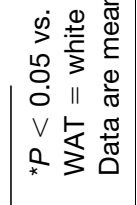 \\
\hline
\end{tabular}




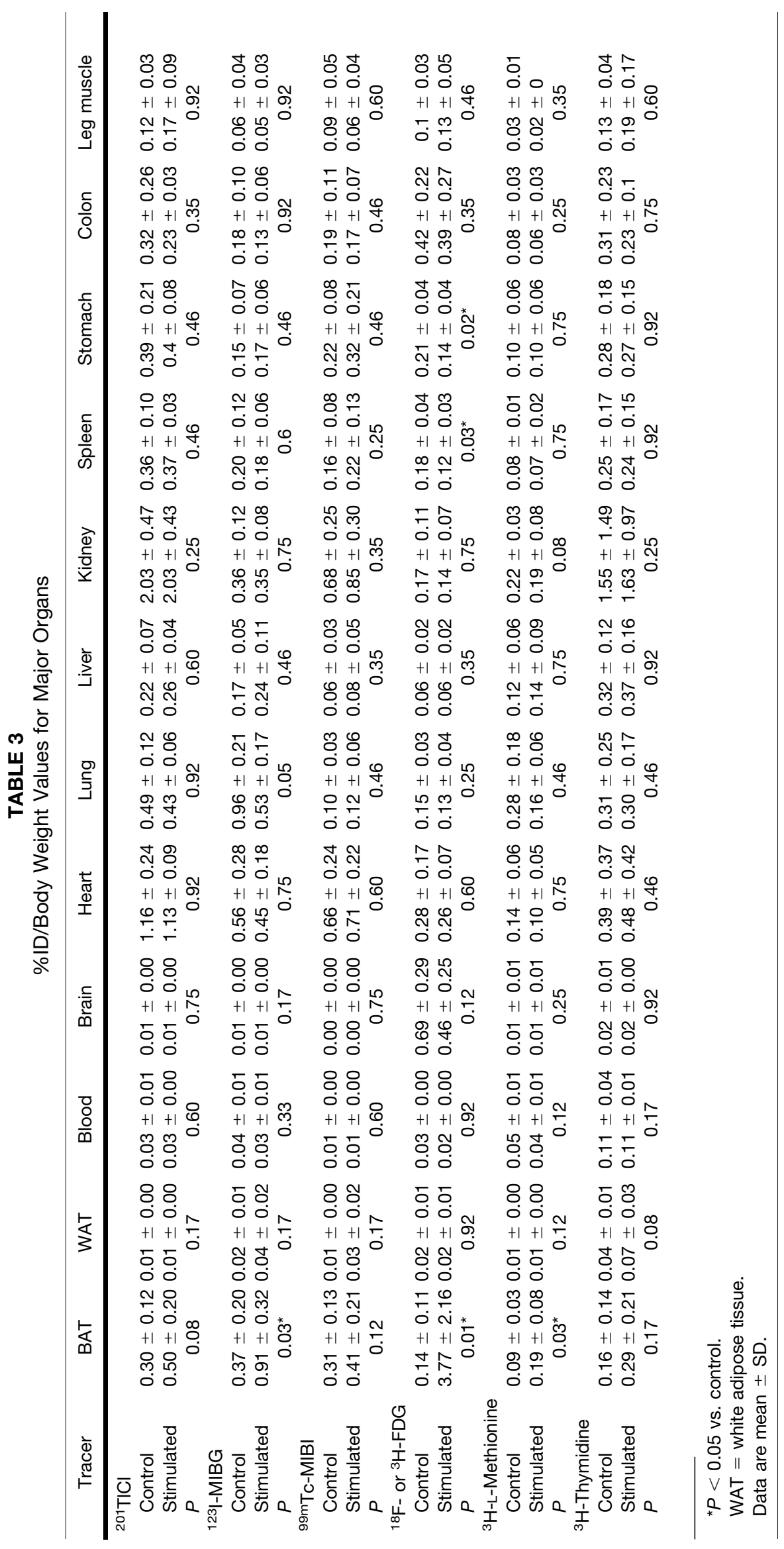


Immunohistolocalization of UCP-1, Glut-1, and NET in BAT under nonstimulated and cold-stimulated conditions is shown in Figure 3. Dense cytoplasmic staining with UCP-1 was seen in BAT under both the control and the cold-stimulated conditions. Partly because of the markedly reduced lipid content in cold-stimulated BAT, the total cytoplasmic UCP-1 content appeared somewhat higher. Staining with GLUT-1 was faint in control BAT cells, whereas dense staining was seen evenly in the plasma membrane of cold-stimulated BAT cells and blood vessels. NET expression was also increased by exposure to cold. However, the distribution of NET was different from that of GLUT-1: Dense staining was seen along the capillary blood vessels, and mild staining was seen in the plasma membrane of BAT cells.

\section{DISCUSSION}

Information about body temperature, feeding status, and body energy reserve is coordinated in the ventromedial hypothalamic nucleus, which signals the release of norepinephrine via the sympathetic nervous system and initiates triglyceride breakdown in BAT primarily via $\beta 3$-adrenergic receptors. Stimulation of norepinephrine induces increased lipoprotein lipase, leading to the release of triglycerides and
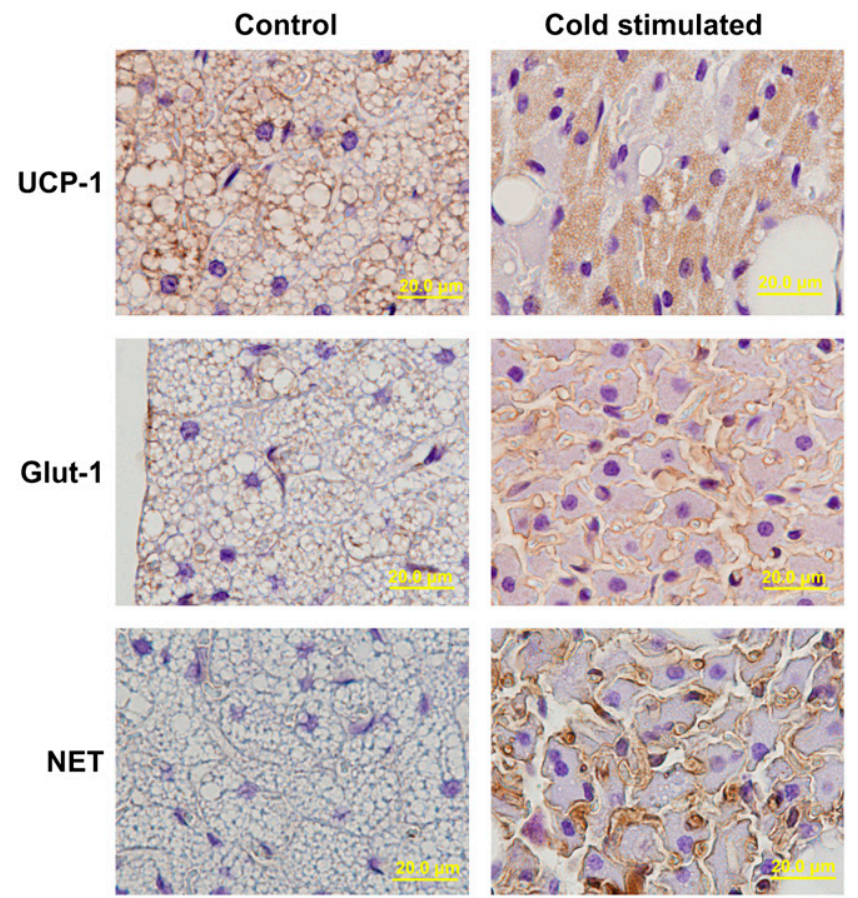

FIGURE 3. Immunohistolocalization of UCP-1, Glut-1, and NET in BAT under control and cold-stimulated conditions. Dense cytoplasmic staining of UCP-1 is seen in BAT under both conditions. Staining of GLUT-1 is faint under control conditions, whereas definite staining is evenly distributed in plasma membrane of BAT under stimulated conditions. NET is also stimulated by cold; however, its distribution is different from that of GLUT-1. Dense staining is seen along with capillary blood vessel, and mild staining was seen in plasma membrane of BAT cells. fatty acids, which are both the acute substrate for thermogenesis and the regulators of UCP-1 activity (1). Triglycerides stored in BAT itself are a major resource for thermogenesis, as well as for glucose to cope with stimulation by acute cold. Our results show that the number of lipid vacuoles in BAT was substantially decreased after stimulation by cold (Figs. 1B and 1C). Thomson et al. reported that a reduction in fat droplet size in BAT was observed during the first $3 \mathrm{~d}$ of stimulation by cold and was followed by a gradual increase in size over a 6-wk period (15). In our study, BAT changed to a reddish and dark color after exposure to cold (Fig. 1A). This change may be due to both an increase in blood flow and a decrease in the lipid content of BAT.

After stimulation by cold, statistically significant $(P<$ $0.05)$ increases in radiotracer uptake were seen for ${ }^{123} \mathrm{I}$ MIBG, ${ }^{18} \mathrm{~F}$ - or ${ }^{3} \mathrm{H}-\mathrm{FDG}$, and ${ }^{3} \mathrm{H}-\mathrm{L}-\mathrm{methionine}$. The increase in uptake of ${ }^{201} \mathrm{TlCl}$, compared with control, was also significant when normalized to blood. In humans, the major structures surrounding BAT are muscle and blood vessels. Thus, activity in these nearby tissues is highly relevant to clinical imaging results. Detection of abnormal signals requires comparison with these normal structures.

We have provided data on tracer uptake normalized to both blood and muscle, as well as the absolute \%ID/body weight data. Because normalization to the blood background value also reduces any variability that may be due to differences in injection technique, we have emphasized the tracer uptake normalized by blood in this study.

A cold-stimulated increase in uptake of ${ }^{201} \mathrm{TlCl}$ may result from increased blood flow in BAT. Astrup et al. reported that BAT blood flow was increased by ephedrine stimulation and resulted in a high temperature in the interscapular subcutaneous region (16). This result of Astrup et al. suggests that blood flow in BAT may be increased by changes in metabolic activity induced not only by ephedrine but also by exposure to cold.

With ${ }^{18} \mathrm{~F}$ - or ${ }^{3} \mathrm{H}$-FDG, BAT uptake was quite low under control conditions and increased significantly (26.5-fold; $P<0.01)$ after stimulation by cold. This increase could be interpreted as a result of increased glucose uptake and consumption by BAT, as is also supported by the Glut-1 immunohistostaining result indicating that the facilitated glucose transporter was stimulated and contributed to increased uptake of ${ }^{18} \mathrm{~F}-$ or ${ }^{3} \mathrm{H}-\mathrm{FDG}$. These findings are consistent with previous reports $(17,18)$.

${ }^{123} \mathrm{I}-\mathrm{MIBG}$ is known to accumulate in the sympathetic nerve terminals, and BAT activation is substantially controlled by the sympathetic nervous system (1). Some reports suggest activation of the sympathetic nervous system is an essential step to activating BAT. Okuyama et al. reported high ${ }^{123}$ I-MIBG uptake in BAT (8). Our results showed that the increase in ${ }^{123}$ I-MIBG uptake was 3.25-fold higher after exposure to cold than under control conditions. This increase is somewhat higher than that of ${ }^{201} \mathrm{TlCl}$, implying that factors other than blood flow are likely to be involved. 
The results of immunohistostaining with an anti-NET antibody revealed that expression of the NET protein was induced in capillary vessels after cold activation and might facilitate uptake of norepinephrine into BAT. Wakayama et al. reported that NET is expressed in the endothelial cells of capillary vessels in the brain (19). NET also appears to be expressed in the endothelial cells of capillary vessels in BAT. We believe this phenomenon indicates that the peripheral sympathetic nervous system in BAT is stimulated by a signal from the hypothalamus under cold conditions. As a result, the activity of NET increases and results in increased uptake of ${ }^{123}$ I-MIBG. ${ }^{123}$ I-MIBG uptake and NET expression have previously been reported to correlate well (20). This process may be related to the enlargement of vascular vessels and increase in blood flow that occur in stimulated BAT.

${ }^{99 \mathrm{~m}} \mathrm{Tc}-\mathrm{MIBI}$ is a tracer that was originally designed to investigate myocardial perfusion. This tracer is taken up in normal mitochondria via passive or active transport into the cell (21-23), and images of this tracer represent the function of the mitochondria, their density, and tracer delivery, especially with longer uptake times. Fukuchi et al. reported that high ${ }^{99 \mathrm{~m} T c-M I B I}$ uptake is seen in the cervical region of children, especially in winter (9), and suggested that ${ }^{99 \mathrm{~m} T c-M I B I}$ uptake into BAT was stimulated by cold temperatures. No significantly increased uptake was observed in our cold-activation model for ${ }^{99 \mathrm{~m}} \mathrm{Tc}-\mathrm{MIBI}$. This discrepancy may partly be due to the mechanism of BAT activation. Chronic exposure to cold, such as during a seasonal change in climate, leads to an increase in the mass of BAT. From our results, uptake of ${ }^{99 \mathrm{~m} T c-M I B I}$ into BAT relative to blood was the highest among the 6 tracers we evaluated in this study, even in the nonstimulated state, and BAT was the organ with the third highest uptake in the body, less than uptake in the kidney and heart (Table 1). These findings indicate that BAT may likely be visualized on clinical ${ }^{99 \mathrm{~m} T c-M I B I}$ scans even in the nonstimulated state, especially in children, who have relatively more BAT than do adults. If the total amount of BAT increases, the incidence of high uptake seen on a scintigram may also increase. Another explanation is that ${ }^{99 \mathrm{~m}} \mathrm{Tc}-\mathrm{MIBI}$ is known as an agent with high lipophilicity (24) and may wash out when cold activation produces an acute decrease in the fat content of BAT. As shown in Figure 1, the total lipid content in BAT was dramatically reduced after acute exposure to cold. Increased ${ }^{99 \mathrm{~m} T c-M I B I}$ uptake in BAT may have been offset by rapid lipid clearance.

Significantly increased uptake of ${ }^{3} \mathrm{H}-\mathrm{L}$-methionine was observed after stimulation by cold in our experiment. BAT has glycolytic capabilities, as well as enzymes involved in amino acid metabolism (25). Uptake and release of several amino acids in BAT was evaluated by Lopez-Soriano et al., who studied arteriovenous differences in vivo (13). Our results indicate that increased ${ }^{3} \mathrm{H}$-L-methionine metabolism in BAT is also measurable with radiolabeled tracers and may potentially be visualized as high uptake in ${ }^{3} \mathrm{H}$-L-methionine PET studies.
Although not statistically significant $(P=0.17)$, increased tracer uptake after stimulation by cold was also seen in the animal groups that received ${ }^{3} \mathrm{H}$-thymidine. The blood-normalized uptake ratio of activation/nonactivation was 2.17 for ${ }^{3} \mathrm{H}$-thymidine-similar to that of ${ }^{201} \mathrm{TlCl}$ (2.04). A longer observation time may have increased the differences in ${ }^{3} \mathrm{H}$-thymidine uptake.

Some significant changes in radiotracer uptake relative to blood after exposure to cold were seen in other major organs. For example, changes occurred in the brain, stomach, and spleen (decreased uptake with ${ }^{18} \mathrm{~F}$ - or ${ }^{3} \mathrm{H}-\mathrm{FDG}$ ), white adipose tissue (increased uptake with ${ }^{3} \mathrm{H}$-L-methionine), and kidney (increased uptake with ${ }^{99 \mathrm{~m} T c-M I B I) . ~ W e ~ c a n n o t ~}$ fully explain these findings. A possible explanation is decreased uptake of ${ }^{18} \mathrm{~F}$ - or ${ }^{3} \mathrm{H}$-FDG in the stomach because of a decrease in gastric peristalsis via cold-stimulated activation of the sympathetic nervous system. Lower brain ${ }^{18} \mathrm{~F}$ - or ${ }^{3} \mathrm{H}$-FDG uptake in cold-stimulated animals may partly be due to decreased sensory stimulation during exposure to cold. The $P$ values for most organs were relatively high for all tracers, suggesting that BAT is the major organ influenced by exposure to cold (Table 1).

UCP-1 is expressed only in BAT and is a key protein that enables mitochondrial oxidation not by coupling with adenosine triphosphate synthesis but rather by promoting heat generation (1). Initially, we anticipated some differences in UCP-1 expression between the control group and the cold-stimulated group. However, UCP-1 expression was seen under both control and stimulated conditions, suggesting that UCP-1 is likely constitutively expressed in BAT. Thus, BAT expresses UCP-1 protein under both nonstimulated and stimulated conditions. Substantial morphologic changes make it difficult to assess small changes in absolute UCP-1 expression. In this study, we did not perform a quantitative analysis of this protein. It is therefore difficult to say whether a true change in the expression level of this protein occurs with stimulation by cold. We believe that this result assists our understanding of how UCP-1 is expressed under control conditions. Staining with UCP-1 is also important by providing clear evidence that what we were evaluating was truly BAT.

To our knowledge, no previous reports have described cold-stimulated changes in the uptake of various clinical radiotracers by BAT under identical conditions. Our results indicate that high tracer uptake would be expected to be observed in clinical SPECT studies using ${ }^{201} \mathrm{TlCl}$ or ${ }^{123} \mathrm{I}-$ MIBG and in PET studies using ${ }^{11} \mathrm{C}$-methionine or ${ }^{18} \mathrm{~F}$-FDG. It is important to know the behavior of various clinical tracers in BAT - especially when stimulated by a low-temperature environment-for correct interpretation of nuclear medicine images.

\section{CONCLUSION}

BAT uptake (\%ID/blood) of ${ }^{18} \mathrm{~F}$ - or ${ }^{3} \mathrm{H}-\mathrm{FDG},{ }^{123} \mathrm{I}-\mathrm{MIBG}$, ${ }^{201} \mathrm{TlCl}$, and ${ }^{3} \mathrm{H}-\mathrm{L}-\mathrm{methionine}$ was significantly higher after 
exposure to cold than in the control state. The greatest increase in tracer uptake was for ${ }^{18} \mathrm{~F}$ - and ${ }^{3} \mathrm{H}-\mathrm{FDG}$. Increased uptake of ${ }^{201} \mathrm{TlCl}$ in cold-stimulated BAT suggests that blood flow in BAT is increased by exposure to cold, but the greater increased uptake for ${ }^{123} \mathrm{I}-\mathrm{MIBG},{ }^{3} \mathrm{H}-\mathrm{L}-\mathrm{methionine}$, and ${ }^{18} \mathrm{~F}$ - or ${ }^{3} \mathrm{H}$-FDG $(P<0.05)$ and the immunohistostaining findings indicate that other factors in addition to blood flow (e.g., increased metabolism) are involved in cold-stimulated BAT activation. Knowledge of the distribution of commonly used clinical radiotracers in BAT, normally and after exposure to cold, will help avoid errors in the interpretation of various nuclear medicine studies.

\section{ACKNOWLEDGMENT}

We thank Judy Buchanan for her assistance in the preparation of the manuscript.

\section{REFERENCES}

1. Cannon B, Nedergaard J. Brown adipose tissue: function and physiological significance. Physiol Rev. 2004;84:277-359.

2. Weber WA. Brown adipose tissue and nuclear medicine imaging. J Nucl Med. 2004;45:1101-1103.

3. Cohade C, Mourtzikos KA, Wahl RL. "USA-Fat": prevalence is related to ambient outdoor temperature-evaluation with ${ }^{18}$ F-FDG PET/CT. J Nucl Med. 2003;44:1267-1270.

4. Cohade C, Osman M, Pannu HK, Wahl RL. Uptake in supraclavicular area fat ("USA-Fat"): description on ${ }^{18}$ F-FDG PET/CT. J Nucl Med. 2003;44:170-176.

5. Hany TF, Gharehpapagh E, Kamel EM, Buck A, Himms-Hagen J, von Schulthess GK. Brown adipose tissue: a factor to consider in symmetrical tracer uptake in the neck and upper chest region. Eur J Nucl Med Mol Imaging. 2002; 29:1393-1398.

6. Garcia CA, Van Nostrand D, Atkins F, et al. Reduction of brown fat 2-deoxy-2[F-18]fluoro-D-glucose uptake by controlling environmental temperature prior to positron emission tomography scan. Mol Imaging Biol. 2006;8:24-29.

7. Okuyama C, Ushijima Y, Kubota T, et al. ${ }^{123}$ I-Metaiodobenzylguanidine uptake in the nape of the neck of children: likely visualization of brown adipose tissue. J Nucl Med. 2003;44:1421-1425.

8. Okuyama C, Sakane N, Yoshida T, et al. ${ }^{123} \mathrm{I}$ - or ${ }^{125} \mathrm{I}$-metaiodobenzylguanidine visualization of brown adipose tissue. J Nucl Med. 2002;43:1234-1240.
9. Fukuchi K, Ono Y, Ishikawa Y, Okada Y, Hayashida K, Ishida Y. Harness-shaped distribution in pediatric Tc-99m tetrofosmin scintigraphy. Clin Nucl Med. 2002;27:303.

10. Higuchi T, Kinuya S, Taki J, et al. Brown adipose tissue: evaluation with ${ }^{201} \mathrm{Tl}$ and ${ }^{99 \mathrm{~m}}$ Tc-sestamibi dual-tracer SPECT. Ann Nucl Med. 2004;18:547-549.

11. Tatsumi M, Engles JM, Ishimori T, Nicely O, Cohade C, Wahl RL. Intense ${ }^{18} \mathrm{~F}-$ FDG uptake in brown fat can be reduced pharmacologically. J Nucl Med. 2004; 45:1189-1193.

12. Baba S, Tatsumi M, Ishimori T, Lilien DL, Engles JM, Wahl RL. Effect of nicotine and ephedrine on the accumulation of ${ }^{18} \mathrm{~F}-\mathrm{FDG}$ in brown adipose tissue. J Nucl Med. 2007;48:981-986.

13. Lopez-Soriano FJ, Fernandez-Lopez JA, Mampel T, Villarroya F, Iglesias R, Alemany M. Amino acid and glucose uptake by rat brown adipose tissue: effect of cold-exposure and acclimation. Biochem J. 1988;252:843-849.

14. Hsu SM, Raine L, Fanger H. Use of avidin-biotin-peroxidase complex (ABC) in immunoperoxidase techniques: a comparison between $\mathrm{ABC}$ and unlabeled antibody (PAP) procedures. J Histochem Cytochem. 1981;29:577-580.

15. Thomsom JF, Habeck DA, Nance SL, Beetham KL. Ultrastructural and biochemical changes in brown fat in cold-exposed rats. J Cell Biol. 1969;41:312334.

16. Astrup A, Bulow J, Christensen NJ, Madsen J. Ephedrine-induced thermogenesis in man: no role for interscapular brown adipose tissue. Clin Sci (Lond). 1984; 66:179-186.

17. Nikami H, Shimizu Y, Endoh D, Yano H, Saito M. Cold exposure increases glucose utilization and glucose transporter expression in brown adipose tissue. Biochem Biophys Res Commun. 1992;185:1078-1082.

18. Shimizu Y, Nikami H, Saito M. Sympathetic activation of glucose utilization in brown adipose tissue in rats. J Biochem (Tokyo). 1991;110:688-692.

19. Wakayama K, Ohtsuki S, Takanaga H, Hosoya K, Terasaki T. Localization of norepinephrine and serotonin transporter in mouse brain capillary endothelial cells. Neurosci Res. 2002;44:173-180.

20. Altmann A, Kissel M, Zitzmann S, et al. Increased MIBG uptake after transfer of the human norepinephrine transporter gene in rat hepatoma. J Nucl Med. 2003;44: 973-980.

21. de Jong M, Bernard BF, Breeman WA, et al. Comparison of uptake of ${ }^{99 \mathrm{~m} T c-}$ MIBI, ${ }^{99 \mathrm{~m}} \mathrm{Tc}$-tetrofosmin and ${ }^{99 \mathrm{~m}} \mathrm{Tc}-\mathrm{Q} 12$ into human breast cancer cell lines. Eur J Nucl Med. 1996;23:1361-1366.

22. Arbab AS, Koizumi K, Toyama K, Araki T. Uptake of technetium-99mtetrofosmin, technetium-99m-MIBI and thallium-201 in tumor cell lines. J Nucl Med. 1996;37:1551-1556.

23. Maublant JC, Zhang Z, Rapp M, Ollier M, Michelot J, Veyre A. In vitro uptake of technetium-99m-teboroxime in carcinoma cell lines and normal cells: comparison with technetium-99m-sestamibi and thallium-201. J Nucl Med. 1993;34: 1949-1952.

24. Mariani G, Molea N, La Civita L, Porciello G, Lazzeri E, Ferri C. Scintigraphic findings on ${ }^{99 \mathrm{~m} T c-M D P,}{ }^{99 m} \mathrm{Tc}$-sestamibi and ${ }^{99 \mathrm{~m}} \mathrm{Tc}-\mathrm{HMPAO}$ images in Gaucher's disease. Eur J Nucl Med. 1996;23:466-470.

25. Lopez-Soriano FJ, Alemany M. Activities of enzymes of amino acid metabolism in rat brown adipose tissue. Biochem Int. 1986;12:471-478. 